\title{
Ultrafast Laser Surface Structuring of Intraocular Lens Polymers
}

\author{
Johannes HEBERLE ${ }^{* 1,2}$, Florian KLÄMPFL ${ }^{1}$, Ilya ALEXEEV ${ }^{1,2}$ and Michael SCHMIDT ${ }^{1,2}$ \\ ${ }^{*}$ Chair of Photonic Technologies, University of Erlangen-Nürnberg, Paul-Gordan-Str. 3, \\ 91052 Erlangen, Germany \\ E-mail: johannes.heberle@lpt.uni-erlangen.de \\ ${ }^{2}$ School of Advanced Optical Technologies, University of Erlangen-Nürnberg, Paul-Gordan-Str. 6, \\ 91052 Erlangen, Germany
}

\begin{abstract}
Ultrafast laser material processing is an attractive approach for surface structuring of medical polymers used for intraocular lenses. To achieve best results in terms of efficiency and quality, the wavelength and pulse energy dependence on the ablation behavior of such materials has to be studied. Ablation thresholds have been determined for a polymer with different dyes which is used for intraocular lens (IOL) manufacturing. Furthermore, the ablation efficiency, achievable precision and surface roughness have been quantified to optimize the parameters for an efficient intraocular lens manufacturing process by surface microstructuring.
\end{abstract}

DOI:10.2961/jlmn.2013.01.0011

Keywords: picosecond, ultrashort pulses, ablation, wavelength dependent, polymer, intraocular lens, structuring, surface roughness

\section{Introduction}

Various diseases of the human eye are treated in ophthalmology by the implantation of intraocular lenses. These lenses can either replace the natural eye lens like in cataract surgeries or they can be implanted in addition to the human lens to treat hyperopia or myopia if a refractive surgery (LASIK) is not possible. Such intraocular lenses consist either of conventional PMMA or modern hydrophobic or hydrophilic acrylate polymers. By addition of an UV- or blue-absorbing dye, the retina is protected from hazardous radiation. To provide best healing after surgery, modern lenses can be folded and inserted into the eye through a very small incision [1].

Presbyopia is the decrease of the natural eye lens elasticity and leads to the loss of the eye's accommodation ability. This disease affects mostly elder people and is usually corrected by the use of glasses. Either, glasses can correct only far or near vision, depending on the kind of glasses. Alternatively, very expensive progressive glasses can be used.

Another promising approach is an implantation of an intraocular lens with two focal points. With such a lens, two images are created on the retina: one for far and one for near vision. The human brain is able to filter out the correct image depending in which distance the person wants to see sharply. Two focal points of one lens are realized by combining diffraction and refraction. So-called apodized ramp-like structures lead to diffraction of $30 \%$ of the incident light into the first focal point. Their size is on the order of $200 \mu \mathrm{m}$ in length and $0.2-2.0 \mu \mathrm{m}$ in height. The remaining light is refracted into the second focal point (Fig. 1). These microstructures are conventionally produced by diamond turning or by injection-molding [2]. However, these techniques suffer from certain disadvantages. In case of turning, the processing time is very high and the reproducibility is low due to tool wear. Injec- tion-molded lenses possess low flexibility and bad biocompatibility [3].

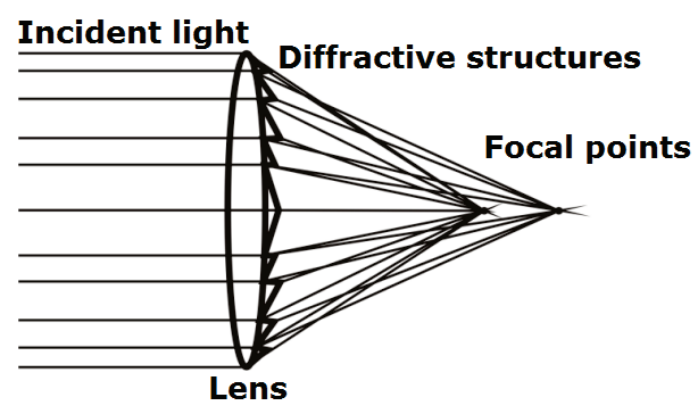

Fig. 1: Imaging principle of a multifocal intraocular lens

An alternative method is the use of ultrafast laser material processing for generation of such micro-scale surface structures. This approach has gained significant attention in the last years as various applications of ultrashort laser pulses, especially for medical applications like shaping biomedical polymers led to promising results [4].

To establish such a process for manufacturing intraocular lenses, investigations on the laser ablation behavior of intraocular lens materials have to be performed. As of now the most promising laser tool for IOL material removal can be high power picosecond lasers since they possess the required pulse energy levels and are suitable for industrial environment. They can produce multiple wavelengths via harmonic generation with the second and the third harmonics, being in the visible and UV spectral ranges correspondingly. The IOL materials to be investigated are commercially available copolymers of PHEMA (80\%) and PMMA (19\%) with two different absorbers (1\%) for UV- (Contaflex stan) or blue light (Contaflex yellow) with the transmission spectra for each material shown in Fig. 2. 


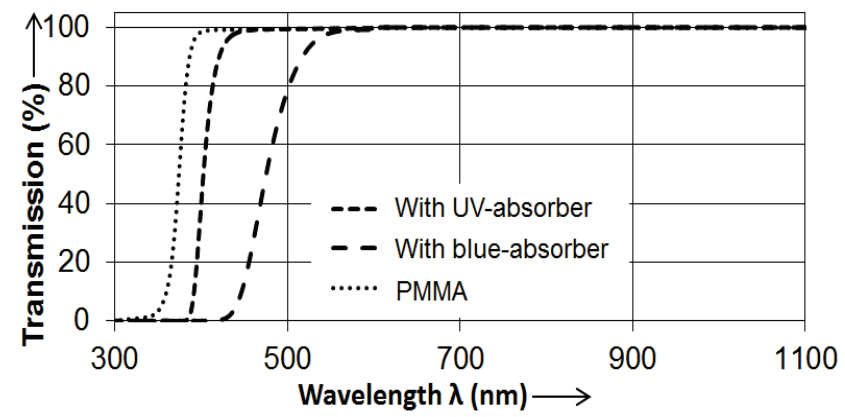

Fig. 2: Transmission spectra of the investigated IOLmaterials. PMMA transmission spectrum is shown for comparison (sample thickness $\mathrm{d}=3 \mathrm{~mm}$ ).

Although the IOL materials appear to be nontransparent for the light below $400 \mathrm{~nm}$, the actual penetration depth in the UV region can be on the order of $100 \mu \mathrm{m}$. To achieve the required precision the laser matter interaction process must occur in the nonlinear regime via multiphoton absorption. In this case the ablation behavior becomes strongly wavelength dependent and must be thoroughly investigated. In this paper we present results of experimental determination of ablation thresholds, ablation rates, and the resulting surface roughness since these parameters will eventually determine feasibility of the proposed IOL lens manufacturing approach.

\section{Experimental setup}

In the presented work we used a Nd:YVO 10 picosecond laser system Fuego from Time-Bandwidth. The output laser power is wavelength dependent with maximum levels being $50 \mathrm{~W}, 25 \mathrm{~W}, 14 \mathrm{~W}$ for primary $(1064 \mathrm{~nm})$, second $(532 \mathrm{~nm})$, and third $(355 \mathrm{~nm})$ harmonics correspondingly. The pulse repetition rate can be continuously varied between a single pulse and $8.2 \mathrm{MHz}$. The beam quality $\mathrm{M}^{2}$ is specified at 1.3 in both directions. The laser beam (all wavelength) is focused with a fused silica $75 \mathrm{~mm}$ focal length lens resulting in focal spot sizes $w_{0}\left(1 / \mathrm{e}^{2}\right)$ of $15 \mu \mathrm{m}$, $9 \mu \mathrm{m}$, and $5 \mu \mathrm{m}$ correspondingly. These values are calculated later on by applying Eq. (1) on the results from experiments on single pulse ablation. The samples are placed on 3D linear translation stages (Fig. 3).

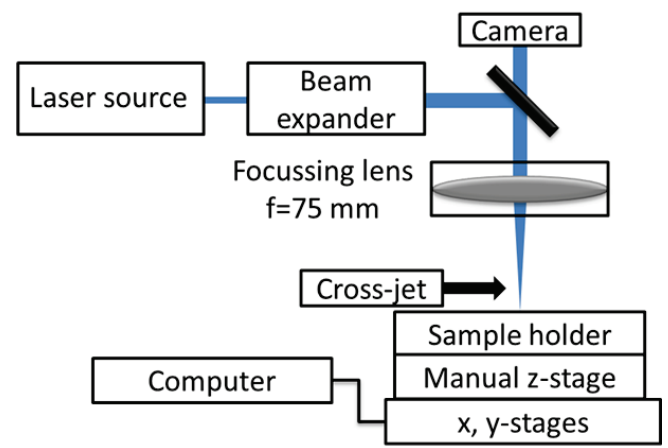

Fig. 3: Experimental setup

The x-y-positioners are motorized stages (Aerotech Inc.) that allow a precise control of feed rate and pulse overlap. The manual z-stage is used to align samples surface with the beam waist. An air flow of about $p=2$ bar pressure above the sample, the cross-jet, prevents redeposi- tion of already ablated material on the sample surface. The ablated volume and the resulting surface roughness were measured with a laser scanning microscope (Keyence, VK9700) to determine process efficiency and quality of the produced surfaces.

\section{Determination of ablation thresholds}

For ultrafast laser ablation of polymers a well-defined threshold is predicted. The determination of ablation thresholds is based on the method by Liu [7]. The diameter $D$ of an ablated area is related to the ablation threshold $F_{t h}$ :

$$
D^{2}=2 w_{0}^{2} \ln \left(F_{0}\right)-2 w_{0}^{2} \ln \left(F_{t h}\right)
$$

$D$ : Diameter of the ablated area

$w_{0}$ : Focal spot radius

$F_{0}$ : Peak fluence

$F_{t h}$ : Ablation threshold fluence

For this purpose, the polymer surface is ablated by single pulses with different pulse energies. The diameter of the modified area can be identified easily by light microscopy as shown in Fig 4. As the spots are not perfectly circular the diameter is measured two times perpendicular to each other. From these two values the mean value is calculated.

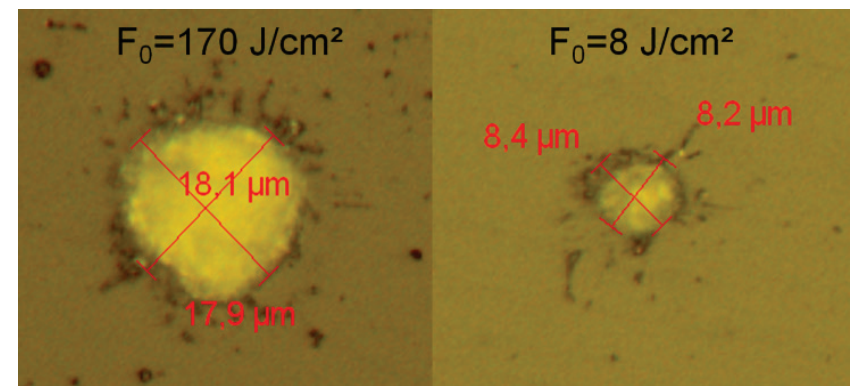

Fig. 4: Single laser pulse ablated area with $\lambda=355 \mathrm{~nm}$

The ablation threshold $F_{t h}$ and the respective focal spot size $w_{0}$ can be determined as described in [9]. First of all, the squared diameter of the ablated area is plotted versus the logarithm of the pulse energy. The gradient of a best fit straight line allows us to calculate the focal spot size $w_{0}$. By identifying that size, the pulse energy depending peak fluence $F_{0}$ can be determined from the measured laser power. Finally, the squared diameter of the ablated area can be plotted semi-logarithmic as a function of the peak fluence. This was performed at different wavelengths and is shown exemplary for Contaflex yellow in Figure 5.

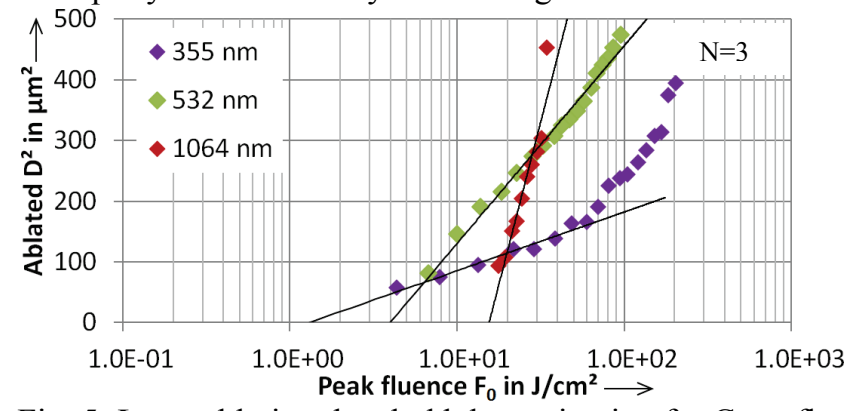

Fig. 5: Laser ablation threshold determination for Contaflex yellow at different wavelengths. 
The intersection between the regression line and the xaxis represents graphically the ablation threshold $F_{t h}$, valid for that specific wavelength and pulse duration. The gradient of the line is proportional to the $1 / \mathrm{e}^{2}$-diameter. The figure shows also a sudden increase of the ablated area more than predicted in Eq. (1) for $F_{0}>60 \mathrm{~J} / \mathrm{cm}^{2}$. This can be explained by chemical decomposition of the polymer. Hot electron diffusion into the material might also be a reason [9]. However, this fluence range is not of interest for precision processing as it will be performed at low fluence and high repetition rates to reduce material damage.

Table 1 shows the identified ablation thresholds depending on the laser wavelength for both materials at fluence $F_{0}<50 \mathrm{~J} / \mathrm{cm}^{2}$.

Table 1 Determined ablation thresholds

\begin{tabular}{ccc}
\hline Material & Contaflex yellow & Contaflex stan \\
\hline $1064 \mathrm{~nm}$ & $12.9 \pm 2.5 \mathrm{~J} / \mathrm{cm}^{2}$ & $13.9 \pm 2.5 \mathrm{~J} / \mathrm{cm}^{2}$ \\
$532 \mathrm{~nm}$ & $4.0 \pm 0.8 \mathrm{~J} / \mathrm{cm}^{2}$ & $5.5 \pm 0.8 \mathrm{~J} / \mathrm{cm}^{2}$ \\
$355 \mathrm{~nm}$ & $1.4 \pm 0.2 \mathrm{~J} / \mathrm{cm}^{2}$ & $1.2 \pm 0.2 \mathrm{~J} / \mathrm{cm}^{2}$ \\
\hline
\end{tabular}

It can be clearly seen, the difference in the ablation thresholds of both materials is negligible at the absorbing wavelength $355 \mathrm{~nm}$. At transparent wavelengths like $532 \mathrm{~nm}$ and $1064 \mathrm{~nm}$ a slightly higher threshold for the material with UV-absorber is identified. This leads to the assumption that mainly a multiphoton absorption results in photothermal and photochemical ablation [6]. The blueabsorber, however, can absorb slightly more energy, leading to a lower threshold. In the UV-region both dyes can absorb photons having no different effect on the ablation. The main result for structuring applications of such materials is that both can be processed in the same way. Due to their similar response to UV-irradiation, especially short wavelengths are preferred at processing. This was also confirmed by differential scanning calorimetry (DSC) measurements which showed equal values for heat capacity and dissociation energy for both polymers.

Furthermore, the thresholds are strongly increasing with longer wavelength. The value rises from UV to NIR by one magnitude approximately. This curve can be interpreted as an ablation by multiphoton absorption. Electrons in a dielectric medium can only absorb photons with more energy than the band gap of the medium. To be able to absorb photons with lower energy, multiple photons have to interact with the electron at the same time. This can be achieved by very high intensities leading to a nonlinear relation between applied intensity and absorption probability [15].

Comparison with previous publicated ablation thresholds for PMMA shows results of the same magnitude. Baudach et.al. [8] and Nam et.al. [16] reported thresholds of $F_{t h}=2.6 \mathrm{~J} / \mathrm{cm}^{2}$ and $F_{t h}=2.4 \mathrm{~J} / \mathrm{cm}^{2}$ respectively for $\tau_{p}=150 \mathrm{fs}$ and $800 \mathrm{~nm}$ wavelength. The higher values of the thresholds at $1064 \mathrm{~nm}$ presented in our work could be explained by the difference of the laser parameters. The multiphoton absorption process, which is assumed to be present at picosecond processing, is strongly dependent on the intensity and on the amount of photons to be absorbed to overcome the band gab of the material. The usage of femtosecond laser pulses in the previous work results in two magnitudes higher intensities at same fluence and provides due to the higher photon energy the possibility to absorb three instead of four photons [16]. Thus, the absorption length of the material is reduced non-linearly, so the incident energy can be absorbed in a thinner surface layer. As less energy is needed to ablate the same area due to the smaller underlying volume the ablation thresholds decreases with shorter wavelength and pulse duration.

\section{Ablation depth}

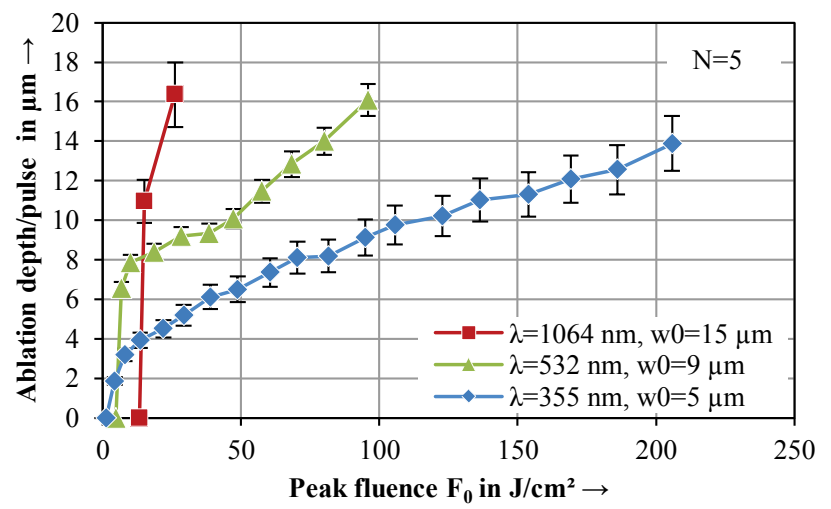

Fig. 6: Ablation depth depending on the peak fluence $F_{0}$.

In Fig. 6 ablation depths are plotted depending on the peak fluence of the incident pulses. In the experiments mean ablated depth was measured after the sample were irradiated with ten pulses at constant pulse frequency of $\mathrm{f}_{\mathrm{p}}=1 \mathrm{kHz}$ to rule out heat accumulation effects [8].

All three displayed wavelengths show similar behavior. If the fluence is high enough to start a multiphoton absorption energy deposition into the material becomes wavelength independent. The free electrons of the ionized molecules can easily absorb any photon energies and conduct it to the surrounding. This is also known as the avalanche ionization [17].

The wavelength dependent sudden drop of the ablation depth at low fluencies results from the well-defined ablation thresholds showed before. To ablate a layer with certain thickness over the whole area, an overlap of the single pulses and high enough fluence above the threshold are required.

However, the step height of the desired diffractive structures is much smaller than the achievable minimal ablation depth of about $2 \mu \mathrm{m}$ for $355 \mathrm{~nm}, 6 \mu \mathrm{m}$ for $532 \mathrm{~nm}$ and $12 \mu \mathrm{m}$ for $1064 \mathrm{~nm}$. To overcome this issue an ablation of an additional layer with high precision in height could be applied to shape the structures on the order of $0.2 \mu \mathrm{m}-$ $2.0 \mu \mathrm{m}$

\section{Surface Roughness}

High quality optics require for high transmission a very high surface flatness. This is usually on the order of $\lambda / 20$ of the visible light. However, as IOL are implanted into the eye and are surrounded by a fluid the effective refractive index difference is reduced. Therefore, a surface flatness of $\lambda / 5$ would be sufficient resulting in a compatible maximum 
roughness of $R_{a}=80 \mathrm{~nm}$. Figure 8 shows the generated surface roughness of the ablated areas. High fluence at $1064 \mathrm{~nm}$ and $532 \mathrm{~nm}$ creates rough edges (Fig. 7) and much debris leading to high roughness. Mechanical stress during the shearing of the ablated layer can cause the edges to shatter [13] and decreases by this the quality of the IOL diffraction. The scattered material remains partially on the surface and increases roughness further.

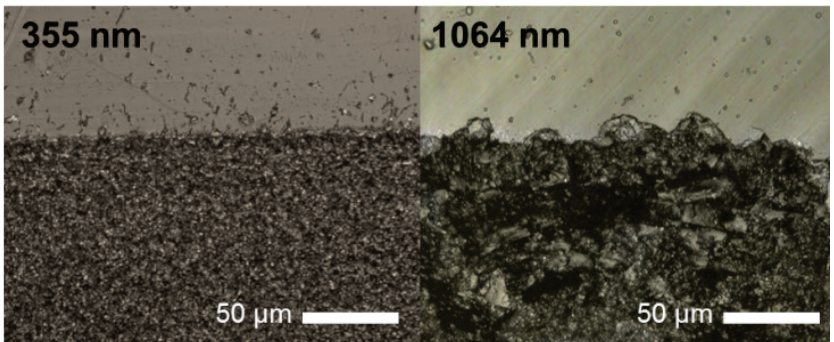

Fig. 7: Microscope image of transition of an processed to an untreated surface $\left(\mathrm{f}_{\mathrm{p}}=1 \mathrm{kHz} ; \mathrm{F}_{0}=13.5 \mathrm{~J} / \mathrm{cm}^{2} ; \tau_{\mathrm{p}}=10 \mathrm{ps}\right.$; $\left.\mathrm{w}_{0,355 \mathrm{~nm}}=5.0 \mu \mathrm{m} ; \mathrm{w}_{0,1064 \mathrm{~nm}}=15.0 \mu \mathrm{m}\right)$

At $355 \mathrm{~nm}$ a more gentle material removal takes place. Clean edges can be produced as well as surface roughness is significantly reduced (Fig. 7). One explanation is the smaller volume ablated per pulse leading to a nearly complete evaporation of the removed material. Also heating of the surrounding area of the irradiated spot can cause increased elasticity [14]. High elasticity prevents brittle fraction and reduce ejection of particles.

Nevertheless, minimum surface roughness was found to be limited to $R_{a}=300 \mathrm{~nm}$ at $355 \mathrm{~nm}$ processing wavelength, $R_{a}=800 \mathrm{~nm}$ and $R_{a}=1300 \mathrm{~nm}$ for $1064 \mathrm{~nm}$.

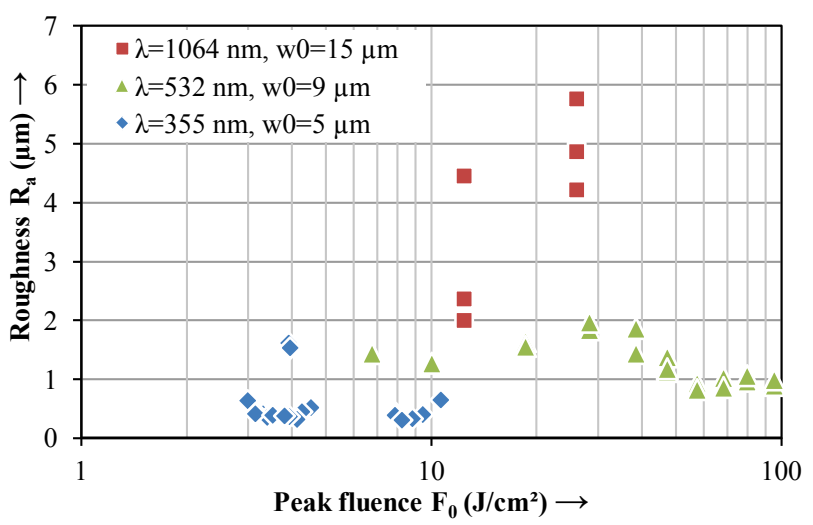

Fig. 8: Summary of wavelength and fluence dependent roughness of the processed surfaces

To achieve best results for surface quality structuring by $355 \mathrm{~nm}$ wavelength was investigated in more detail. Figure 9 shows the roughness after ablation of a layer at respective pulse overlap, pulse energy and pulse frequency. Feed rate can be calculated by using pulse overlap and pulse repetition rate.

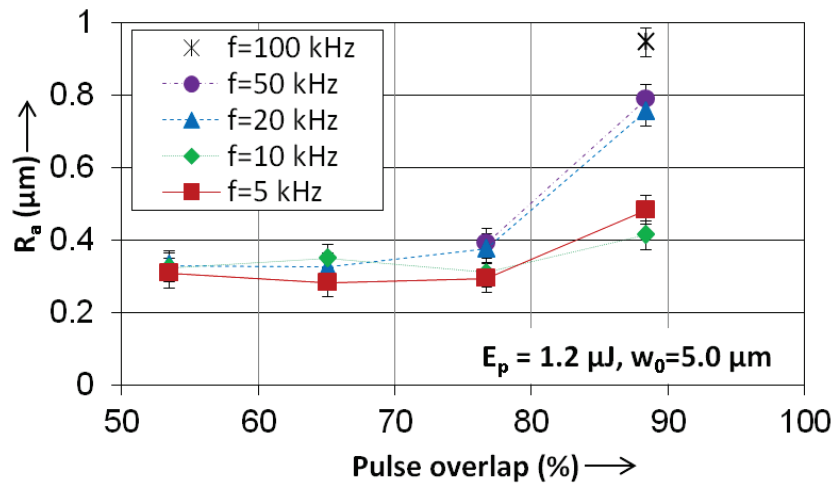

Fig. 9: Detailed roughness results on processing at $355 \mathrm{~nm}$

Pulse overlap $\Omega<50 \%$ leads to irregular ablation results as the ablated spots cannot form a closed surface. It was found that pulse overlap of $\Omega=50 \%$ - $75 \%$ leads to similar surface quality $R_{a} \approx 300 \mathrm{~nm}$ independent from the applied pulse frequency up to $50 \mathrm{kHz}$. At pulse overlap of $\Omega>75 \%$ an increase in roughness is detected, even stronger for higher pulse frequencies. Both behaviors can be explained by a heat accumulation effect. The heating of the surrounding area at single pulse ablation remains at high pulse rates until the next pulse and is attenuated further. This effect can be strong enough to create large heat affected zones and melting of the neighboring material leading to high surface roughness. This however counteracts the cold ablation process by ultrashort pulsed lasers and has to be prevented.

\section{Conclusion and Outlook}

Investigations on laser ablation of IOL materials have been performed. Wavelength dependent ablation showed an increase of the threshold for longer wavelength, however, was nearly the same for both analyzed dyed polymers. The ablation depth increases with rising fluence but has a sudden drop at low fluence depending on the wavelength. This means a single material removal step by pulsed laser irradiation is limited to a certain minimal achievable ablation depth of a few micrometers.

The quality of the processed surfaces was evaluated by its roughness and the edge quality. It was found that also minimal achievable roughness is limited depending on the wavelength and increases with longer wavelengths. Longer wavelengths which are transparent to the material showed material shatter at the edges. This may be caused by high temperature gradients and high mechanical stress during ablation. For transparent wavelengths only in the ablated area energy is deposited and heats the material strongly until ablation. For short wavelengths also linear absorption is present which also heats the surrounding area of the ablation zone. As elasticity is high with increasing polymer temperature the brittleness is reduced leading to smooth and straight edges after the shear off of the layer.

To summarize our results we determined $355 \mathrm{~nm}$ as processing wavelength to be the most promising especially for surface structuring applications. Low ablation thresholds enable high accuracy material removal by processing at low pulse energies. Highest precision can be reached in terms of lateral precision by the focal spot size and vertical precision by the minimal ablation depth. Furthermore, the 
roughness was observed to be low compared to processing at other wavelengths.

Detailed investigations on the process parameters at $355 \mathrm{~nm}$ processing revealed strong dependence on heat accumulation of the surface quality. With pulse overlap of approximately $\Omega=50 \%$ and low pulse frequency low surface roughness can be achieved, however, leads to long processing time. Therefore, a trade-off between quality and processing time has to be made in form of a proposed twostep process. First a rough shape of the lens is produced with fast processing time by high repetition rates, feed rates and pulse energies. The following second step is used for precision processing of the diffractive structures, reduction of surface roughness and minimal material damage.

However, as the short wavelength can directly break the bonds of the polymers and creates free radicals which may change the chemical composition of the IOL material. Therefore, biocompatibility tests have to be carried out to ensure that the laser processed material is still suitable for implants.

\section{Acknowledgements}

We would like to thank the German Federal Ministry of Education and Research (BMBF) for funding the project "A-03: Erarbeitung technologischer Grundlagen für die spätere Entwicklung und Herstellung von optimierten phaken Intraokularlinsen - Teilprojekt 2: Oberflächenstrukturierung transparenter Biomaterialien mittels Laserstrahlung" (promotional reference: 01EX1011C) as well as our project partner the HumanOptics AG for providing the IOL-samples and the Erlangen Graduate School in Advanced Optical Technologies (SAOT) for the received support so far.

\section{References}

[1] S.D. McLeod, V. Portney and A. Ting: A dual optic accommodationg lens, Brit. J. Ophthalmol. 87, pp. 1083-1085 (2003)

[2] R. Grolmus: MD-Thesis, University Gießen, (2007)

[3] M.C. Kraff, D. Sanders and H.L. Lieberman: J. Am. Intraocul. Im-plant. Soc. 93, pp. 301-305, (1983)

[4] F. Korte. S. Nolte, B.N. Chichkov, T. Bauer, G. Kamlage, T. Wagner, C. Fallnich and H. Welling: Far-field and near-field material processing with femtosecond laser pulses, Appl. Phy. A, Vol. 69, No. 7 (1999)

[5] A.W. Martinez and E.L. Chalkof: Microfabrication and nanotechnology in stent design, Willey Interdisciplinary Reviews: Nanomedicine and Nanobiotechnology, Vol. 3, pp. 256-268 (2011)

[6] E. Spyratou, I. Asproudis, D. Tsoutsi, C. Bacharis, K. Moutsouris, M. Makropoulou and A.A. Serafetinides: UV laser ablation of intraocular lenses SEM and AFM microscopy examination of the biomaterial surface, Applied Surface Science 256, pp. 2539-2545 (2010)

[7] J.M. Liu: Simple technique for measurement of pulsed Gaussian-beam spot sizes, Opt. Let. Vol. 7 No. 5 pp. 196 (1982)

[8] S. Baudach, J. Bonse, J. Krüger and W. Kautek: Ultrashort pulse laser ablation of polycarbonate and polymethylmethacrylate, Appl. Surf. Sci. 154-155, pp. 555-560 (2000)
[9] J. Bonse, J.M. Wrobel, J. Krüger and W. Kautek: Ultrashort-pulse laser ablation of indium phosphide in air, Appl. Phys. A 72, pp. 89-94 (2001)

[10] E. Spyratou, M. Makropoulou and A. A. Serafetinides: Study of visible and mid-infrared laser ablation mechanism of PMMA and intraocular lenses: experimental and theoretical results, Lasers Med Sci, 23: pp. 179-188 (2008)

[11] D. Bäuerle: Laser Processing and Chemistry, Springer-Verlag Berlin Heidelberg, (2011)

[12] N. Arnold, N. Bityurin and D. Bäuerle: Laser-induced thermal degradation and ablation of polymers: bulk model, Appl. Surf. Sci., Vol. 138-139, pp. 212-217 (1999)

[13] N Bityurin, B.S. Luk'yanchuk, M.H. Hong and T.C. Chong: Models for Laser Ablation of Polymers, Chem. Rev. 2003

[14] P.J. Flory: Principles of polymer chemistry, pp. 442, Ithaca, New York (1995)

[15] I. Miyamoto, K. Cvecek and M. Schmidt: Evaluation of nonlinear absorptivity in internal modification of bulk glass by ultrashort laser pulses, Opt. Exp. Vol. 19, pp. 10714-10727 (2011)

[16] J.R. Nam, C.H. Kim, S.C. Jeong, K.S. Lim, H.M. Kim, S.-J. Jeon and B.R. Cho: Measurement of twophoton absorption coefficient of dye molecules doped in polymer thin films based on ultrafast laser ablation, Chem. Phys. Let. 427, pp. 210-214 (2006)

[17] W.L. Smith, J.H. Bechtel and N. Bloembergen: Picosecond laser-induced breakdown at 5321 and $3547 \AA$ : Observation of frequency-dependent behavior, Phys. Rev. B., Vol. 15, No. 8, pp. 4039-4055 (1977)

(Received: June 13, 2012, Accepted: December 18, 2012) 